\title{
LA MÚSICA COMO ACTIVIDAD DE EXTENSIÓN UNIVERSITARIA DURANTE EL FRANQUISMO: LA CÁTEDRA DE MÚSICA FRANCISCO SALINAS
}

\author{
Music as a University Extension Activity during Franco's Regime: \\ Francisco Salinas Chair of Music
}

\author{
Sara González Gómez \\ sara.gonzalez@uib.es \\ Universitat de les Illes Balears. España \\ Llorenç Gelabert Gual \\ llorens.gelabert@uib.cat \\ Universitat de les Illes Balears. España
}

Fecha de recepción: 04/04/2020

Fecha de aceptación: 20/04/2020

Resumen: Durante el franquismo, la relación entre música y universidad se produce siempre ligada a actividades de extensión universitaria y difusión cultural. A partir de la década de los cincuenta, estas actividades de carácter musical comienzan a canalizarse dentro de las universidades a través de las cátedras especiales de música. El presente artículo se centra en el estudio del proceso de creación, reglamentación y funcionamiento de estas cátedras y presenta una aproximación a las principales actividades desarrolladas por la cátedra Francisco Salinas de Salamanca durante el periodo de 1967/68 a 1974/75. El objetivo principal que se persigue es averiguar si estas cátedras ayudaron o no al regreso de la música a los espacios universitarios, así como a la posterior introducción de la musicología dentro de los planes de estudio.

Palabras clave: música; universidad; cátedras especiales; franquismo; cátedra de música Francisco Salinas.

Abstract: During Franco's regime, the relationship between music and university always develops closely connected with university extension courses and cultural diffusion. From the fifties, these musical activities begin to make their way into universities through special Chairs of music. This paper focuses on the study of the creation process, regulation and operation of these Chairs and adopts an 
approach to the main activities developed by Francisco Salinas Chair in Salamanca during the period $1967 / 68$ to $1974 / 75$. The main objective pursued is whether or not these Chairs helped music return to university spaces as well as the subsequent introduction of musicology in the curriculum.

Keywords: music; university; special Chairs; Franco's regime; Francisco Salinas Chair.

SUMARIO. 1. Introducción. 2. Música y universidad en España: breve recorrido histórico. 3. La música como actividad de extensión universitaria durante el franquismo. 4. Las cátedras especiales de música en la universidad española del franquismo. 5. Actividades de la cátedra de música «Francisco Salinas» en la Universidad de Salamanca. 6. Conclusiones. 7. Referencias bibliográficas.

\section{INTRODUCCIÓN}

Mediante la Orden de 8 de octubre de $1984^{1}$ se aprobó el plan de estudios de la especialidad de Musicología dentro de la sección de Historia del Arte de la Facultad de Geografía e Historia de la Universidad de Oviedo. Posteriormente se crearon especialidades en Salamanca, Autónoma de Barcelona, La Laguna de Tenerife y Granada, entre otras (Casares, 1998). Este hecho resituaba a la música dentro de la universidad, sin necesidad de retrotraernos al esplendor del medievo, en el estatus de materia científica que otrora representó. La presión ejercida por el colectivo de expertos en musicología, desde dentro y fuera de las propias universidades, y la emergente demanda social tanto de formación como de información musical, ligada a la difusión de los medios de comunicación, fueron algunos de los aspectos que más influyeron de cara a este reconocimiento (Medina, 1986, pp. 14-15). Asimismo, jugaron un importante papel en la vuelta de la música a la universidad tanto las escuelas de formación de maestros y su vinculación con las nuevas metodologías musicales (Oriol, 2012, pp. 13-42; Alonso y Rodríguez, 1999) como las cátedras especiales de música creadas a partir de la década de los cincuenta del siglo Xx. Capítulo aparte merecen, en cambio, los conservatorios, que, si bien siendo las instituciones que debían recoger el testigo formativo e investigador de las extinguidas cátedras de finales del XVIII, su tarea se centró, eminentemente, en la formación en solfa y práctica instrumental hasta nuestros días (Marías, 1999, pp. 76-79).

De entre los diversos aspectos mencionados anteriormente, el presente artículo se centra en el estudio del proceso de creación, reglamentación y funcionamiento de las cátedras especiales de música surgidas durante la segunda mitad del franquismo en diversas universidades de la geografía española. No se dispone hasta el momento de ningún trabajo que haya tenido a estas cátedras como objeto de estudio, motivo por el cual se considera pertinente un primer acercamiento general a las mismas, pues suponen prácticamente la vía exclusiva a través de la cual se hizo efectiva la presencia de la música dentro de las universidades de la época. En

${ }^{1}$ Orden publicada en el BOE núm. 301, de 17 de diciembre de 1984, en el marco de la Ley 14/1970, de 4 de agosto, General de Educación y Financiamiento de la Reforma Educativa. 
consecuencia, el objetivo general de este trabajo será descubrir sus características, funciones y competencias y realizar una aproximación, de forma específica, a las principales actividades desarrolladas por la cátedra Francisco Salinas de la Universidad de Salamanca.

El artículo se ha organizado en cuatro grandes apartados: un primero en el que se efectúa una breve síntesis histórica sobre la presencia de la música en la universidad; en segundo lugar, se aportan unas sucintas pinceladas sobre la música como actividad de extensión universitaria y difusión cultural, tal y como fue contemplada su presencia dentro de la universidad del franquismo; un tercer punto está dedicado a la creación de las cátedras especiales de música -desde la Cátedra Manuel de Falla en la Universidad de Madrid durante el año 1952 hasta la aparición de la Cátedra Francisco Salinas en la Universidad de Salamanca en 1968-, analizando sus reglamentos y recorriendo aspectos tales como las razones para su creación, funciones o cometidos de las mismas, organización de las enseñanzas, dirección, dotaciones y financiación; finalmente, se presenta un listado detallado de las actuaciones desarrolladas por la cátedra Francisco Salinas de Salamanca entre los cursos $1967 / 68$ y 1974/75, como muestra concreta de la actividad real y efectiva de estas resurgidas cátedras.

\section{MÚSICA Y UNIVERSIDAD EN ESPAÑA: BREVE RECORRIDO HISTÓRICO}

La presencia de la música en los planes de estudio, tanto en la vertiente práctica o interpretativa como en la teórica, se remonta al mismo origen de las universidades europeas. Constituía, junto a las matemáticas, la aritmética y otras disciplinas, una materia esencial del quadrivium medieval. Destacan universidades como la de París, Orleans, Oxford, Cambridge, Bolonia o Padua, y, por sus aportaciones en forma de escritos y tratados sobre teoría musical, figuras como Jerónimo de Moravia, Aribo Scholasticus, Robert Grosseteste, Walter Odington, Vittorino de Feltre, Johannes Gallicus o Adelardo de Bath.

La primera universidad española, y una de las primeras constituidas en Europa, fue la Universidad de Salamanca, fundada por autorización real de Alfonso IX en el año 1218. Esta misma universidad, por disposición de Alfonso X el Sabio, creó la primera cátedra de música europea en el año 1254 (García, 1991; García, 1999). Este acontecimiento marcó el inicio de un periodo de apogeo y afloramiento de investigaciones y aportaciones musicales, tanto a nivel de especulación teórica como de actividad musical. En Salamanca, como en la mayoría de universidades europeas, los estudiantes de artes liberales debían estudiar música obligatoriamente junto al resto de materias del quadrivium. Además, era común participar en los coros eclesiásticos auspiciados por las mismas universidades o practicar algún instrumento. Esta presencia consta en una Bula de Clemente $V$ del año 1313, por la cual la música 
aparece incluida entre las materias enseñadas por maestros y doctores salmantinos, con referencias explícitas al magistri in musica en el año 1355. Cabe también destacar el famoso tratado Ars musica de Juan Gil de Zamora, relacionado con la Universidad de Salamanca. Dicho tratado aportaba elementos musicales enfocados desde la matemática, aspectos relacionados con la notación, modos, etc. (Carpenter, 1958, cit. en Tur, 1992, pp. 78-97). A lo largo de los siglos XIV y XV siguió proliferando la producción de tratados sobre teoría musical. Es de obligada mención la figura del musicólogo español y catedrático de la Universidad de Salamanca, Bartolomé Ramos de Pareja (1440- ), y sus novedosas aportaciones en el ámbito tonal a través de su obra De musica Tractatus, sive Musica practica, publicada en Bolonia en el año 1482 (Wolf, 1944).

Durante el Renacimiento, la música continúa siendo objeto de estudios científicos diversos, en un intento de intelectualización del arte en sí. De nuevo, la Universidad de Salamanca se erige como sede de enseñanza, apreciada por su espíritu humanista, progresista y tolerante (Sarget, 2000, p. 121). Emerge en el siglo XVı la figura de Francisco de Salinas (1513-1590), teórico musical y a la postre uno de los personajes más ilustres de la historia de la música en España. Destacaron sus aportaciones en el ámbito de la música y su relación con la matemática, el temperamento tonal y el valor de la práctica musical, situándola en el mismo rango jerárquico que la teoría y como parte inmanente a la misma. En su tratado de armonía y teoría rítmica De Musica libri septem ${ }^{2}$, Salinas defiende la idea que media hora de trabajo con música especulativa o teórica debía verse complementada con media hora de práctica, ya fuese en la práctica del órgano o del canto llano. Cabe destacar, en otro orden de cosas, que la creación de la cátedra Francisco Salinas en la década de los sesenta del siglo pasado, y objeto de este estudio, constituyó un merecido tributo al prolífico legado del humanista salmantino y fue el germen, junto a otras iniciativas, de la posterior reincorporación de los estudios musicológicos en la universidad.

Resulta remarcable en este mismo siglo la presencia y prestigio de la música en la Universidad de Alcalá, a pesar de no contar con una cátedra específica, mediante figuras destacadas como Pedro Ciruelo y Juan Bermudo, autor este último de Arte Tripharia, un tratado sobre canto llano, música mensural y técnica de instrumentos de tecla (Martín, sin fecha). A las grandes aportaciones de Salinas y Bermudo se debe añadir la figura de fray Tomás de Santamaría y su Arte de tañer fantasía, obra en la que presenta un sistema de digitación para la vihuela e instrumentos de tecla y un análisis de las ornamentaciones musicales de la época. Aparecen de forma recurrente multitud de tratados teórico-musicales vinculados a la técnica del canto llano y su uso litúrgico por toda la península. Destacan, entre otros, Arte de canto Ilano

2 Con motivo del quinto centenario del nacimiento de Francisco de Salinas, Ediciones Universidad de Salamanca publicó, en el año 2013, la obra completa De Musica libri septem, con la supervisión y aportaciones de Amaya García Pérez y Bernardo García-Bernalt Alonso. 
de autor anónimo; Ars Musicorum de Guillermo de Podio, en Valencia; Lux videntis de Bartolomé de Molina, en Valladolid; Arte de canto Ilano y contrapunto y canto de órgano con proporciones y modos y Intonaciones nuevamente corregidas según uso de los modernos que hoy cantan y entonan en la Iglesia Romana de Gonzalo Martínez de Vizcargui, en Burgos; o, Libro de música práctica de Francisco Tovar, en Barcelona (Martín, 2005).

Por tanto, a la largo de toda la época renacentista la música mantuvo una notoria presencia en los estudios de la Universidad, tanto en el ámbito de la enseñanza como de la investigación. Esta situación se mantuvo a lo largo de los siglos XVII y XVIII. Lo acreditan la gran cantidad de estudios teórico-prácticos que se fomentan tanto desde las propias universidades como desde las capillas de música catedralicias. Marcelino Menéndez Pelayo, historiador y estudioso del ideario estético en España, reconoce la riqueza y abundancia de tratados musicales españoles a lo largo de esta época. Destaca además en sus aportaciones el fuerte vínculo que entonces se establece entre música, educación y formación, tanto en las escuelas episcopales y monásticas como en las universidades. En cuanto a tratados y publicaciones, proliferan aquellos referentes a música religiosa y música profana, artes del canto llano, canto de órgano y contrapunto y artes de vihuela o de guitarra, entre otros (Menéndez, 1974). Al frente de la cátedra de música a lo largo del siglo XVII destacan figuras reconocidas como Sebastián de Vivanco, Roque Martínez, Juan Berjón, Antonio de Castro, Juan de Torres y Roca y Diego Verdugo. En la Universidad de Alcalá es destacable la presencia del reconocido teórico y organista Andrés Lorente a mediados de siglo.

En el XVIII fueron catedráticos de música en Salamanca Tomás de Mizieces, Antonio de Yanguas, Juan Antonio de Aragüés, Antonio de Argüelles, Gabriel Perlines y Manuel Doyagüe. A mediados de este siglo, la situación de la universidad distaba mucho del esplendor y repercusión de siglos anteriores. El número de alumnos se redujo considerablemente y del prestigio de antaño, con figuras como Francisco de Vitoria, Fray Luis de León o Francisco de Salinas, se pasó a un marcado periodo de decadencia tanto en el ámbito académico como en la organización administrativa (García-Bernalt, 2014; García-Bernalt, 2015).

En el año 1772 se suprime, por Provisión Real, la Cátedra de Música de la Universidad de Salamanca (García Fraile, 2004, p. 44). En el nuevo plan propuesto se recalcó la continuidad de la música práctica a través de la orquesta universitaria, y la teórica a través de los catedráticos de matemáticas. Aunque fuera así a corto plazo, tras la supresión de la Cátedra de Música de la Universidad de Salamanca los espacios formativo-musicales en España se sumergieron en un gradual letargo científico, de negativas consecuencias, y que perduró a lo largo de dos siglos.

Contrariamente a la situación en España cabe destacar el emergente proceso de culturización e impulso de la investigación musical que se promovió entonces desde las universidades del resto de Europa, destacando principalmente las francesas, 
italianas e inglesas. Si bien los estudios musicológicos se reincorporaron a la universidad española en la década de los ochenta del siglo pasado, el largo periodo de inacción nos situó en un peldaño inferior respecto al resto de países de Europa. Inmediatamente antes de esa reintegración de la música a la universidad durante la etapa democrática, se van a crear las cátedras especiales de música sobre las cuales trataremos de arrojar algo más de luz a lo largo de las siguientes páginas.

\section{LA MÚSICA COMO ACTIVIDAD DE EXTENSIÓN UNIVERSITARIA DURANTE EL FRANQUISMO}

El régimen franquista, en cuanto a referentes musicales, optó por un argumentario noventayochista dirigido a la recuperación de los signos de identidad nacionales, con especial influencia del folklore andaluz, y al impulso de la zarzuela, de corte castizo y costumbrista. Se pretendía con ello transmitir una imagen homogénea de la actividad cultural y musical en España, alejada del modelo político y territorial planteado por la República. Bien que las aportaciones de autores reconocidos internacionalmente como Falla, Albéniz o Turina fueron utilizadas como ejemplificación del compromiso con la renovación estética por parte del régimen, el resto de representantes de la vanguardia musical en España, más cercanos al ideario republicano en la mayoría de los casos, marcharon todos al exilio. Otros autores que permanecieron en España optaron por líneas estilísticas más convencionales y cercanas al tardorromantisismo (Piñeiro, 2013).

El nivel de intervencionismo por parte del régimen en el campo de la creación musical, en cuanto a directrices estéticas se refiere, no alcanzó altas cotas -sí aplicadas en otras disciplinas-. La principal razón se encuentra en el empuje nacionalista de autores de principios de siglo xx, a partir del resurgimiento de un estilo neoclásico con un papel preponderante de elementos castizos. Esta inercia de exacerbación patriótica repercutirá de forma evidente en un claro retraso estético respecto a la vanguardia europea, que contrasta con el uso propagandístico llevado a cabo por parte del régimen, con una cúpula de poder mucho más proclive a estos registros (Muñiz, 1998).

En cuanto a la enseñanza en las escuelas, los cambios fueron relevantes en tanto en cuanto quedó diferenciada la educación entre ambos sexos, tanto en ubicación como en contenidos, haciendo patente así la afinidad del régimen con los principios pedagógicos del catolicismo más reaccionario. La Sección Femenina fue la institución encargada de transmitir los valores y la formación a las mujeres durante la mayor parte del periodo franquista. Entre las materias que se impartían en dicha sección encontramos la formación musical en solfeo y canto, que consistía en una reducida introducción al lenguaje musical y la interpretación de un repertorio de canciones propias del folklore castizo. En relación a la formación en los 
conservatorios, esta sigue dirigida única y exclusivamente a la formación de músicos. En 1942 se crea una normativa específica referente a estas instituciones, casi inexistente y ambigua hasta entonces. Surgen así tres categorías en los Conservatorios Oficiales de Música y Declamación: Superiores, Profesionales y Elementales. Esta reglamentación se verá aún más definida y concretada en contenidos a través del Decreto 2618/1966, de 20 de septiembre, el conocido como Plan 66. Del mismo modo establecerá las bases para la creación de nuevos conservatorios en todo el estado español, no necesariamente superiores. Esta pequeña estructura legal creada en torno a los estudios musicales no se vio en absoluto ligada al sistema educativo general y de ninguna manera se convirtió en un vínculo en la implantación de la enseñanza de la música en las escuelas. Asimismo, las titulaciones que se obtenían en los conservatorios no tenían un reconocimiento jurídico claro y explícito, lo que contribuía aún más al desprestigio social y también retributivo imperante en materia musical.

La relación entre música y universidad durante este periodo se produjo siempre ligada a actividades de extensión universitaria. Es decir, la música no formaba parte de los planes de estudio generales ni encontramos estudios propios de musicología en su oferta formativa, relegando su valor y aportación a espacios como acompañamiento en actos o acontecimientos académicos de carácter ceremonioso o conciertos y audiciones musicales a modo de reclamo en el marco de las llamadas actividades de extensión cultural. A partir de la década de los cincuenta, este tipo de actividades universitarias empiezan a gestionarse a través de las denominadas cátedras especiales de música. Por ello, se ha considerado imprescindible dedicar las siguientes líneas a explicar, sucintamente, la evolución y el sentido de la extensión universitaria antes y durante la época que acota este trabajo, pasando posteriormente al análisis detallado de dichas cátedras.

La extensión universitaria o cultural como fenómeno educativo hunde sus raíces en los planteamientos y alcances de la Institución Libre de Enseñanza (ILE) y tiene como referentes internacionales el modelo anglosajón y las universidades populares de origen francés. Desde sus primeras referencias acotadas a programas de educación formal de adultos en la Gran Bretaña de finales del siglo XVIII, pasando por la labor desarrollada en ateneos, sociedades económicas de amigos del país, escuelas populares, cursos de verano o universidades populares, entre otras muchas instituciones, hasta la actual concepción de la extensión universitaria como uno de los "radios de acción» presentes en todas las universidades, se han dado numerosas y muy variadas experiencias que hacen de este concepto un término enormemente diversificado.

Superado en España el primer tercio del siglo xx, época fructífera en el terreno científico y de extensión cultural, estalla la guerra civil y la posterior época de régimen dictatorial. A partir de este momento, las actividades de extensión cultural adquieren unos claros tintes propagandísticos. Pero lo cierto es que el fortalecimiento 
de la función social de la universidad mediante la utilización de la proyección de su quehacer hacia la sociedad, poniendo en práctica los programas de extensión universitaria y difusión cultural, fue una tarea poco cultivada en la vida universitaria de la primera mitad del franquismo. A pesar de que ya en el artículo 13 de la Ley de 29 de julio de 1943 sobre ordenación de la universidad española se establecían los órganos mediante los cuales las universidades desarrollarían sus funciones primordiales, entre ellos el Secretariado de Publicaciones, Intercambio Científico y Extensión Universitaria (SPICEU) ${ }^{3}$, la puesta en marcha de actividades de extensión se produjo de forma tímida y pausada. Solo a partir de los años cincuenta, y especialmente en décadas posteriores, comenzaron a darse algunos tímidos proyectos que entreabrieron levemente el camino.

Así, el propósito de poner el saber universitario al servicio de la sociedad y de hacer de sus problemas tema fundamental de sus preocupaciones, no era una de las prioridades de los dirigentes políticos y universitarios. El hecho de incorporar las tareas de extensión y difusión al quehacer normal de la universidad, pero consideradas como una proyección hacia la comunidad de ese quehacer, como una ampliación de su radio de acción susceptible de permitir la participación en la cultura universitaria de sectores más amplios, no se produjo con frecuencia.

Los programas de extensión cultural de las universidades españolas consistían, por regla general, en el auspicio de conferencias, charlas, escenificaciones teatrales, coros, exposiciones, conciertos, cine-club, emisiones radiofónicas, escuelas sociales, cursos de verano, etc. En algunos casos, estas tareas comprendían también ciertos programas de acción social, como es el caso de los consultorios jurídicos populares.

La mayor parte de estas actividades dependían del referido SPICEU, al cual le correspondían, de acuerdo al artículo 30 de la Ley de 1943, las funciones de difusión de la cultura, enseñanza no propiamente facultativa o profesional e intercambio científico. Entre sus principales competencias nos encontramos con un amplio listado en el que se refería: a) La dirección y el impulso de publicaciones universitarias; b) La dirección de cursos especiales independientes de los propiamente facultativos o profesionales; c) La organización de cursos para extranjeros; d) El intercambio de profesores o alumnos con universidades nacionales o extranjeras; e) La dirección de las cátedras o cursos especiales que, sin estar adscritos a los planes de estudios facultativos o profesionales, existan o se creen en la universidad, cualquier que sea su origen fundacional; f) La edición de programas, temas de cátedra y publicaciones escolares, en relación con el Sindicato Español Universitario (SEU).

EI SPICEU ejercería las funciones expresadas en los apartados c), d) y e) de acuerdo con el Consejo Superior de Investigaciones Científicas (CSIC); y las del apar-

${ }^{3}$ Oficialmente, en todas las universidades de España se crean estos secretariados por Orden de 8 de octubre de 1943. 
tado d), en lo relativo a profesores, de acuerdo con el Servicio Español del Profesorado de Enseñanza Superior de Falange Española Tradicionalista y de las JONS y, en lo que se refiere al alumnado, con el SEU.

En consecuencia, será en ese punto e) relativo a la dirección de cátedras especiales, en colaboración con el CSIC, y concretamente dentro de las secciones de extensión universitaria, donde encontraremos las cátedras especiales de música creadas en distintas universidades de la geografía española.

En términos generales, tal y como afirma Tünnermann Bernheim (2003, pp. 71-72) para las universidades de América Latina, pero perfectamente extrapolable al caso español, las labores de extensión y difusión cultural del momento se caracterizaron por lo siguiente: a) No responder a un programa bien estructurado ni a unos objetivos claramente definidos; b) Tener un carácter marginal, en el sentido de que guardaron poca o ninguna relación con las tareas docentes e investigadoras y con los planes y programas de estudio; c) Responder al tipo de trabajo universitario que demandaba una sociedad clasista, proyectando unas actividades con carácter meramente informativo y ornamental. En definitiva, se demostraba un escaso compromiso de la universidad con todo este tipo de actividades, y con la música en particular.

Pues bien, es dentro de estas coordenadas básicas donde debemos inscribir la relación que se establece entre música y universidad, especialmente durante la segunda mitad del franquismo. La música se convertía en una actividad complementaria o de ampliación de la formación básica y general que seguía el universitario a través de las enseñanzas de la carrera escogida y, por supuesto, siempre con un carácter voluntario. De esta forma, la programación de actividades musicales servía a las universidades como vehículo para canalizar la mayor parte de las labores de las denominadas secciones de extensión universitaria. La música era, en consecuencia, una forma de combinar entretenimiento y atracción cultural de un colectivo de estudiantes universitarios pertenecientes, en su gran mayoría, a una clase social alta.

Básicamente, la música se transmutaba dentro de la universidad del franquismo en una especie de actividad extracurricular, enfocada hacia la extensión cultural $y$, en todo caso, con un carácter secundario dentro de sus funciones y quehaceres esenciales. Pero a pesar de ello, no cabe duda de que sobre ella pivotaron la mayor parte de iniciativas de extensión universitaria implementadas durante la época. La aparición de cátedras especiales de música a lo largo de los años cincuenta y sesenta en varias universidades -las cuales se convertirían en artífices de la programación de conciertos variados, con cierto carácter internacional, conferencias, cursos y ciclos de formación musical- trató quizá de paliar esta situación, la de la falta de conocimientos musicológicos por parte de los universitarios, pero veremos hasta qué punto se consiguió cambiar el decurso de las cosas y cuál fue el verdadero alcance de estas nuevas estructuras a lo largo de las siguientes páginas. 


\section{LAS CÁTEDRAS ESPECIALES DE MÚSICA EN LA UNIVERSIDAD ESPAÑOLA DEL FRANQUISMO}

A partir de la década de los cincuenta comenzaron a aflorar dentro de algunas de las universidades españolas las denominadas como "cátedras de música», en un claro intento por recuperar los espacios perdidos por la música dentro de estas instituciones de formación superior. Muchas de ellas llevaron por denominación el nombre de músicos o compositores de renombre (Felipe Pedrell, Manuel de Falla, Francisco Salinas, Muñiz Toca, Cristóbal de Morales...) y se enfocaron preferentemente hacia el fomento de la cultura musical entre los universitarios y a la difusión del conocimiento de la música a través de la organización de conciertos, audiciones y conferencias.

No es de extrañar que la mayor parte de estas cátedras nacieran durante el periodo ministerial de Joaquín Ruiz-Giménez (1951/56), una etapa caracterizada por el intento de modernización del sistema educativo, de una leve apertura muy pronto cercenada por el propio régimen. Será en el año 1952 cuando se apruebe la creación de la primera de ellas en la Universidad de Madrid, bajo la denominación de Cátedra Manuel de Falla. A ella le seguirán la de Santiago de Compostela, la Cátedra Felipe Pedrell de la Universidad de Barcelona y la Cátedra Manuel de Falla en la Universidad de Granada, todas ellas creadas en el año 1954; la Cátedra Cristóbal de Morales de la Universidad de Sevilla en 1955; ese mismo año se aprueba el reglamento de la Cátedra de Cultura musical Muñiz Toca de la Universidad de Oviedo; y ya a finales de los sesenta (1968) aparecerá la Cátedra de música Francisco Salinas en la Universidad de Salamanca.

Las razones para la creación de estas cátedras quedaron reflejadas en las órdenes mediante las cuales se aprobó su funcionamiento. Así, por ejemplo, en la Orden de 6 de marzo de 1952, por la que se crea la Cátedra Manuel de Falla en la Universidad de Madrid, podemos leer:

En la organización tradicional de la enseñanza universitaria la música ocupó siempre un importante lugar no sólo en su aspecto docente, sino también como medio de formación artística de los estudiantes a la par que instrumento de amenidad entre los alumnos de las diversas facultades. La excesiva especialización de la enseñanza dejó al margen esta cátedra de música que hoy conviene restaurar con arreglo a un plan orgánico. Resulta necesaria y urgente esta restauración, ya que la afición musical entre los universitarios es cada día mayor y conviene, por lo tanto, encauzarla dentro de una cátedra universitaria que atienda a menesteres tan importantes como la unión entre la investigación musical y la enseñanza y el cuidado de cuantas actividades puedan concurrir a la difusión entre los estudiantes de la cultura musical ${ }^{4}$.

${ }^{4}$ Orden de 6 de marzo de 1952 por la que se crea en la Facultad de Filosofía y Letras de la Universidad de Madrid una cátedra de «Música», BOE, n.ㅇ 113 de 22 de abril de 1952. 
La idea de conectar enseñanza e investigación musical, reflejada en las últimas líneas de este extracto, Ilama poderosamente nuestra atención. Es de sobra conocido el letargo y el retraso que a nivel de investigación se sufría en la universidad española durante la primera mitad del franquismo. Pero es cierto también que a partir de la medianía de siglo comenzaron a apreciarse ciertos cambios, aunque todavía tímidos y ni mucho menos generalizados, que apuntaban hacia unas cátedras mucho más activas en las que se desarrollaban interesantes labores de investigación y difusión científica. Aunque la propuesta realizada desde la Universidad de Madrid podía resultar ciertamente utópica, dadas las dificultades y la ausencia de estudios propios de musicología dentro de las instituciones universitarias, resulta cuanto menos esperanzadora. De hecho, las alusiones a labores de investigación se repiten en la mayor parte de los reglamentos aprobados para las cátedras creadas durante la época; así, por ejemplo, en la cátedra de la Universidad de Barcelona se refleja, como su principal cometido, la investigación y difusión de la Historia de la Música.

Obviamente, la universidad necesitaba una modernización de su modelo de investigación, adecuar tanto sus recursos como sus estructuras a los adelantos extranjeros y aportar una nueva orientación al binomio ciencia-técnica; en esencia, una adaptación al modelo humboldtiano de universidad, para lo cual era necesaria la creación de numerosos y bien nutridos laboratorios de experimentación, sostener buenas bibliotecas, bien dotadas y actualizadas, garantizar el intercambio de publicaciones y centros de investigación (Hernández, 2011, p. 229), crear espacios adecuados para albergar discografía y otros recursos audiovisuales y abrir vías a la introducción de nuevas enseñanzas, como es el caso de la música que aquí nos ocupa.

Si acudimos al caso de la Cátedra Francisco Salinas de Salamanca se alegaba también entre sus razones de ser la tradición histórica que los estudios musicológicos habían tenido siempre dentro de esta institución. Recordemos que la Universidad de Salamanca fue la primera de la cultura occidental que incluyó oficialmente la enseñanza de la música en sus programas (Martín, 2005, p. 57), aunque paradójicamente, tal y como vemos, sería de las últimas instituciones en contar con una cátedra de este tipo durante el periodo en estudio.

En cuanto a las funciones o cometidos de estas cátedras, en todos los casos sus reglamentos recogen de forma similar tanto el fomento del estudio, el conocimiento y la enseñanza de la música como la organización de conciertos, audiciones y conferencias para hacer efectiva la primera. Pero repasemos los reglamentos de algunas de estas cátedras para visualizar de forma clara cuáles eran las principales tareas a ellas encomendadas. 
En el caso de la Cátedra Felipe Pedrell de la Universidad de Barcelona ${ }^{5}$ se preveía la concreción en tres ciclos o periodos integrados a lo largo del curso de las actividades desarrolladas, en forma de lecciones y trabajos de seminario dedicados principalmente a: 1) Historia de la música (una época, escuela, género musical, tendencias técnicas, historia de una instrumento o familia instrumental, etc.); 2) Música popular; 3) Biografías de músicos o historia de agrupaciones o escuelas musicales.

El número de conferencias o lecciones de esta cátedra, aparte de los trabajos de seminario, no debía ser superior a veinte ni inferior a diez para cada curso. Asimismo, se establecía que éstas se impartirían preferentemente en los meses de febrero, marzo y abril.

A diferencia de la de Barcelona, en la Cátedra Manuel de Falla de la Universidad de Granada ${ }^{6}$ no se especifican de forma exhaustiva la temática de los cursos, seminarios o conferencias organizadas. Por el contrario, sí se hace alusión entre sus funciones a aspectos tales como la edición de composiciones, obras o estudios de cultura musical de interés artístico, científico o histórico. Asimismo, se le encomienda la protección, como tesoro artístico especialmente vinculado a la ciudad de Granada, de cuanto esté unido a su nombre, así como cualquier otro tipo de actividad que tienda a fomentar el conocimiento de la música entre los escolares o a proyectarla en el ambiente social en que vive la universidad.

Si acudimos al reglamento de la Cátedra Cristóbal de Morales de la Universidad de Sevilla ${ }^{7}$ encontramos, como seña distintiva de la misma, la orientación de las manifestaciones musicales de Sevilla y su distrito universitario, realizando para ello grabaciones de carácter folclórico con objeto de conservar la música popular, así como, igual que sucedía en la de Granada, la edición de composiciones y estudios de cultura musical de interés artístico, científico o histórico. Además, en la normativa aprobada para esta cátedra se hace referencia a la contribución en la formación de coros y agrupaciones musicales universitarias y a su difusión en el país.

En cuanto a la organización de la enseñanza en la cátedra sevillana, se prevé lo siguiente: 1) Un curso general sobre Historia de la música considerada dentro de la Historia de la Cultura y de las Bellas Artes. Esta enseñanza era asimilada a la de "encargo de curso»; 2) Cursos monográficos sobre materias especiales musicales (estas enseñanzas tendrían la consideración de cursos monográficos de Licenciatura o Doctorado para los alumnos de la Facultad de Filosofía y Letras); 3) Conferen-

${ }^{5}$ Orden de 21 de marzo de 1955 por la que se aprueba el reglamento para la Cátedra «Felipe Pedrell» creada en la Facultad de Filosofía y Letras de la Universidad de Barcelona, BOE, n.o 101 de 11 de abril de 1955 .

${ }^{6}$ Orden de 30 de diciembre de 1954 por la que se aprueban las normas para el régimen de la Cátedra «Manuel de Falla» de la Universidad de Granada, BOE, n. 23 de 23 de enero de 1955.

7 Orden de 30 de diciembre de 1954 por la que se aprueba el reglamento de la Cátedra de Música «Cristóbal de Morales» de la Universidad de Sevilla, BOE, n.ㅇ 88 de 29 de marzo de 1955. 
cias sobre autores y obras musicales, impartidas por especialistas; 4) Conciertos y audiciones.

Por su parte, la cátedra de «cultura musical» de la Universidad de Oviedo ${ }^{8}$ contemplaba en su reglamento como principales funciones prácticamente idénticas cuestiones a las ya mencionadas para las anteriores cátedras: fomento del estudio y conocimiento de la música española, organización de lecciones y conferencias de carácter general y preparación de cursos de cultura musical para alumnado universitario. Añadía a éstas, la celebración de conciertos con la Orquesta Sinfónica de Música de Cámara de Asturias y la Orquesta de instrumentos de arco de la Universidad de Oviedo; la recopilación en cinta magnetofónica de canciones y música regional asturiana; y las exhibiciones folclóricas con la intervención de los grupos de coros y danzas de la sección femenina de FET y las JONS.

Entre las funciones o cometidos de algunas de estas cátedras se encontraba la creación de archivos, "discotecas» o bibliotecas mediante las cuales ofrecer al universitario interesado algunos materiales que bien podrían servir para la puesta en marcha de investigaciones originales. Así, por ejemplo, en el reglamento de la Cátedra Felipe Pedrell de Barcelona se apunta lo siguiente:

Se procurará que la cátedra disponga de una abundante discoteca y, cuando sea necesario, de grupos de profesores instrumentista o de coro. Formará también un archivo de diapositivas, fotografías y dibujos, originales musicales, ediciones raras de obras y tratados musicales, así como una biblioteca de obras modernas dedicadas a la Historia de la Música9 .

Cambiando de asunto, la dirección de estas nuevas cátedras se encomendaba a comisiones o patronatos de los que formaría parte siempre el profesor encargado de la cátedra, el Rector de la universidad y el Decano de la Facultad de Filosofía y Letras en cuestión. En algunos casos, a ellos se unirían representantes de otras instituciones, tal es el caso, por ejemplo, de la cátedra de Granada, de la que formaban parte miembros del Ayuntamiento y la Diputación Provincial de Granada y del Conservatorio de Música y Declamación. Lo mismo sucedía en la de Oviedo, en la que, además de todos los representantes señalados, nos encontramos con el Delegado provincial del MIT, el Rector de Seminario Conciliar de Oviedo, los presidentes de las sociedades filarmónicas de Oviedo y Gijón y la Regidora de la Sección Femenina de FET y las JONS.

${ }^{8}$ Orden de 15 de marzo de 1955 por la que se aprueba el reglamento de la Cátedra de «Cultura Musical», creada en la Universidad de Oviedo, BOE, n. 88 de 29 de marzo de 1955.

${ }_{9}$ (Orden de 21 de marzo de 1955 por la que se aprueba el reglamento para la Cátedra «Felipe Pedrell» creada en la Facultad de Filosofía y Letras de la Universidad de Barcelona, BOE, n.ㅇ 101 de 11 de abril de 1955). 
Finalmente, en cuanto a dotaciones y financiación de las cátedras, se reflejaba en la mayoría de los casos la percepción de emolumentos mediante subvenciones del Ministerio de Educación Nacional, la propia universidad, entidades públicas, corporaciones y/o particulares. Además, se obtendrían ingresos mediante la matrícula y los derechos recaudados por las enseñanzas impartidas, así como por los ingresos provenientes de la elaboración y venta de publicaciones.

En definitiva, y haciendo una inicial reflexión sobre el sentido, significado y utilidad de estas cátedras de música creadas durante el franquismo, debemos decir que, a la luz de lo expresado en sus reglamentos, sirvieron para canalizar una serie de actividades relacionadas con el mundo musical y para difundir la cultura musical especialmente entre el alumnado universitario. Asimismo, conectaron universidad, sociedad y ciudad, una tarea del todo necesaria para una universidad que en esos momentos vive prácticamente de espaldas a los círculos que la rodean, dado su carácter elitista y eminentemente tradicional.

Está claro que no eran ni mucho menos los organismos idóneos para la consecución de la introducción de los estudios de musicología dentro de las universidades -algunos especialistas consideran incluso que «no hicieron más que entorpecer la posibilidad del cambio", al convencer a las autoridades académicas y ministeriales que se hacía realmente algo por la música universitaria, pero imposibilitando al mismo tiempo un auténtico inicio del estudio de esta rama del saber (Casares, 1984, p. 157)-, pero tampoco puede negarse su importante labor durante el periodo, aunque enmarcado siempre, y de ahí la importancia que se la ha otorgado en este trabajo, en el campo de la extensión universitaria. De hecho, la opinión de Casares contrasta claramente con la sostenida por Aviñoa (1996, p. 87), para quien, la creación de estas cátedras de difusión cultural de la música, encuadradas dentro del amplio concepto de extensión universitaria que hemos analizado previamente, supone uno de los hitos fundamentales de lo que podríamos denominar como el «retorno» de la música a los ámbitos universitarios.

La necesidad de incoar el cambio, de introducir la musicología dentro de los estudios universitarios y de revalorizar su importancia más allá de las labores meramente de extensión y difusión cultural se hicieron más que evidentes a finales de la década de los sesenta. De hecho, las conclusiones alcanzadas en el «Seminario sobre la música en la Universidad», organizado en Sevilla por la Comisaría General de la Música en octubre de $1969^{10}$, así lo demuestran. Entre otras cosas, en estas jornadas se acordó que en todas las universidades debería crearse una cátedra de

${ }^{10}$ La actividad se enmarca dentro de un Seminario sobre «Problemas actuales de la enseñanza musical» desarrollado en la universidad a finales de la década los sesenta. En concreto, la última sesión, desarrollada en la sala de juntas de la Facultad de Filosofía y Letras de la Universidad de Sevilla, se dedicó a "Música en la Universidad» y en ella participaron «profesores titulares de las cátedras de música de las universidad españolas y destacados especialista, críticos, musicólogos, etc.», $A B C$, sábado 11 de octubre de 1969, Edición de Andalucía, p. 70. 
cultura musical, dependiente del Secretariado de Extensión Universitaria y con el asesoramiento de la Comisaría General de la Música de la Dirección General de Bellas Artes (DGBA). Sería competencia de estas cátedras no solo la difusión cultural sino también la organización y el desempeño de enseñanzas: un curso de Historia de la Música a lo largo del periodo lectivo; comentarios acerca de los compositores, obras e intérpretes que intervengan en conciertos, recitales y grabaciones; cursos monográficos y seminarios a cargo de especialistas; seminarios, coros, grupos instrumentales... ${ }^{11}$.

Pero probablemente una de las cuestiones que mayor peso tendría dentro de las conclusiones alcanzadas en aquel seminario, pronto tenida en cuenta por las autoridades ministeriales, sería la siguiente: «De las enseñanzas de la Cátedra de Arte, que actualmente figura en los cursos de Estudios Comunes de las Facultades de Letras, formará obligatoriamente parte la Historia de la Música». Asimismo, se propuso la creación de departamentos de música, dentro de los cuales podrían realizar las tesis doctorales los alumnos interesados y con ello contribuir a cimentar «la base de una futura labor de investigación en orden a la Historia y Teoría de la Música».

Pero todo esto serán ya desarrollos y alcances posteriores que comenzarán a tomar forma en décadas sucesivas. Volvamos ahora a nuestras cátedras especiales de música y, como muestra de sus actividades, analicemos la labor desempeñada durante el año lectivo por la cátedra de una de las universidades con mayor renombre e historia a sus espaldas, la de la Universidad de Salamanca, para de este modo poder contrastar lo expresado en los reglamentos de creación de las cátedras con la vida real y efectiva que estas tuvieron.

\section{ACTIVIDADES DE LA CÁTEDRA DE MÚSICA «FRANCISCO SALINAS" EN LA UNIVERSIDAD DE SALAMANCA}

Por Orden de 29 de mayo de 1968 se crea en la Universidad de Salamanca la Cátedra "Francisco Salinas», "en homenaje a la memoria del gran músico y humanista español del siglo XVI, que desempeñó su magisterio en la propia Universidad de Salamanca y en cuya obra se resume todo el saber musical de su tiempo» ${ }^{12}$. Francisco Salinas regentó, durante más de veinte años, la cátedra de música en la institución salmantina y falleció en esta ciudad en el año 1590.

La dirección de la nueva cátedra se encomendó al profesor de la Facultad de Filosofía y Letras, Manuel Fernández Álvarez, catedrático de Historia Moderna en la Universidad de Salamanca desde el año 1965. Un profesional que acotó sus

11 Vid. Martín, 2005, p. 69.

12 Orden de 29 de mayo de 1968 por la que se crea en la Universidad de Salamanca la «Cátedra de Música Francisco Salinas», BOE, n.o 159 de 3 de julio de 1968. 
investigaciones, especialmente, al siglo XVI, consiguiendo el Premio Nacional de Historia de España en el año 1985; asimismo, se convirtió en miembro de la Real Academia de Historia en 1987 y en portador de la medalla de oro de la ciudad de Salamanca.

La función principal del director sería la organización, coordinación y desarrollo de conciertos, audiciones y conferencias. También se encargaría de dar los primeros pasos para la creación de una biblioteca y discoteca especializadas. Además, la orden reguladora establecía que dentro de la cátedra se procedería a la edición de publicaciones relacionadas con temas musicales.

Para conocer los primeros pasos de la cátedra, procede hacer referencia a las actividades musicales más relevantes celebradas durante sus primeros años de vida; éstas nos servirán como muestra de la organización y planificación llevada a cabo en los primeros cursos de funcionamiento y como ejemplo de la relevancia que, poco a poco, fueron adquiriendo los conciertos y las audiciones como complemento a diferentes actos académicos, congresos o jornadas universitarias, es decir, como actividades propiamente de extensión universitaria. Al mismo tiempo, podremos comprobar si a través de ella se canalizaron o no actividades de formación musical del universitario y si se desarrollaron o no las mencionadas labores de investigación que como hemos visto se reflejaban en los reglamentos de algunas de estas nuevas cátedras. En este estudio tomaremos como marco cronológico de estudio los cursos que van desde 1967/68, año de su creación, hasta 1974/75, final de la etapa dictatorial.

\section{Curso $1967 / 68^{13}$}

En su primer año de vida la cátedra salmantina organiza 11 conciertos para los cuales cuenta con el coro universitario de la institución (actuando en el Colegio Mayor San Bartolomé en dos ocasiones) y, mayoritariamente, con músicos de diferentes procedencias. Entre ellos destacan, con un concierto de violonchelo ${ }^{14}$ desarrollado en el aula Salinas, la concertista norteamericana Martha Gerschefskl, acompañada al piano por Mr. Mattos; Trilby Lundberg y Ezio Mariani de Amicis con uno de piano y violín y con los mismos instrumentos el de Pedro León y Elisa Ibáñez ${ }^{15}$; y Harry Houdeshel y Edward Mattos, con motivo de las Jornadas Culturales de EE. UU. celebradas en Salamanca, interpretan un concierto de flauta y piano.

13 Información extraía de: AUSA, Memoria del año académico 1967/68, Universidad de Salamanca, 1968. Rev. 1178/39.

${ }^{14}$ El evento fue organizado por la cátedra con la colaboración de la Asociación de antiguos alumnos y amigos de la Universidad (ASUS).

${ }^{15}$ Esta actividad contó con la colaboración de la sección de cultura popular del Ministerio de Información y Turismo (MIT). 
El resto de conciertos son de piano y nos encontramos en ellos a músicos como Marisa Montiel ${ }^{16}$, Margarita Degeneffe de Fuenteseca, que desarrolla dos conciertos, uno con motivo de la celebración de la festividad de Santo Tomás de Aquino y otro en las IV Jornadas de profesores de Derecho procesal; Flor Rodríguez Riego y Josefina Ameigeiras.

A los conciertos señalados se unen varias audiciones musicales celebradas los días 15, 22 y 29 de marzo de 1968 en torno a Beethoven con discos de la Deutsche Grammophon Gesellschaft, acompañadas de comentarios introductorios de P. Coreva, P. Paniagua y Aníbal Sánchez Fraile.

A todas estas actividades se suma la puesta en marcha de lo que se denominó como los "Viernes musicales en la Universidad». Una iniciativa con la que se pretendía acercar la música a un mayor número de público. El propio Balcells informó de este proyecto al director general de enseñanza universitaria, agradeciéndole el apoyo prestado para convertir en realidad la nueva e inusual cátedra que, «con tan buenos ojos, desde el principio animaste ${ }^{17}$.

De la revisión de las actividades desarrolladas por la cátedra de música durante su primer año de vida -o en las que colaboró y se involucró de manera activa-, se desprenden varias conclusiones. En primer lugar, la labor emprendida resultó ciertamente fecunda en un plazo muy breve de tiempo, demostrando una especial implicación y tendencia a la organización de conciertos universitarios. En segundo lugar, resulta interesante constatar el alto número de mujeres que fueron protagonistas de estos actos, reflejo evidente de que las cuestiones de género comenzaban a experimentar importantes cambios dentro de la universidad.

Siguiéndole la pista y avanzando hacia los años de periodo rectoral de Felipe Lucena Conde (1968/72), localizamos nuevas actividades, quizá algo más diversificadas. Veamos la planificación de tareas y actuaciones desarrolladas hasta el final del franquismo:

\section{Curso $1968 / 69^{18}$}

El curso se abre con cuatro audiciones de Música Romántica sobre Beethoven, Schubert, Mendelssohn, Schumann y Chopin, con la colaboración de un grupo universitario compuesto por: Ana Agud, Maribel Mendiburu, Mercedes Ortiz, Alberto Quintana y Baltasar Quart, a cuyo cargo quedaron los correspondientes comentarios.

${ }^{16}$ Se contó, de nuevo, con la colaboración de la sección de cultura popular del MIT.

17 Archivo Histórico de la Universidad de Salamanca (en adelante AUSA), Correspondencia del rector Alfonso Balcells Gorina con el director general de EU del MEN, Salamanca, 12 de marzo de 1968, AC 4314/18.

18 Información extraída de: AUSA, Memoria del año académico 1968/69, Universidad de Salamanca, 1969. Rev. 1178/40: 144-145. 
Asimismo, se desarrolla un recital de guitarra por Arturo Espino Reyes; un concierto del Coro Universitario de Oviedo; y un concierto de piano de Lily Bienvenu.

\section{Curso $1969 / 70^{19}$}

De forma muy similar al curso anterior, en este año se desarrollan cuatro audiciones de música española (ss. XII-XVII), con la colaboración del grupo universitario de la Facultad de Filosofía y Letras compuesto por: Juan Blanco Andray, Fernando García Lima, Encarnación Masot Martínez, M.a Teresa Haro Hernández, Josefa Bernáldez Triviño, M.a Vicenta Julián Gómez, M. a Pilar Sánchez Marcos, M. a Jesús Morán Sánchez, M.a Nieves Montes Rubio, Teresa Zamora de Castro y Josefa Sánchez Mateos, dirigidos por Dámaso García Fraile.

A ellas se suman los conciertos de piano por Ivette Hernández; el concierto "Monumentos de la Música Española» por el Cuarto Renacimiento ofrecido a los miembros del V Coloquio Internacional de Estudios Micénicos; y un concierto de música clásica ofrecido por el cuarteto de Cámara de la RTV Española, patrocinado por el MIT.

Tal y como podemos ver, contrasta el reducido número de actividades programadas para estos dos años académicos con la vida de la cátedra en su primer año así como con el elevado incremento que comenzaremos a observar a partir del próximo curso.

\section{Curso $1970 / 71^{20}$}

Durante el primer trimestre del curso se desarrolla un concierto de los madrigalistas de Praga, en el marco de las "Jornadas Musicales Universitarias", organizadas por la Comisaría General de la Música, de la DGBA; un concierto sobre "La música en Europa (ss. XV-XVI)» por Atrium Musicae; una conferencia-concierto "La Guitarra», por Regino Sainz de la Maza; y un concierto de la Sinfonía de Madrid, dirigido por Vicente Spiteri.

En el segundo trimestre se pone en marcha el curso de formación musical, con audiciones de música desarrolladas por Dámaso G. Fraile; se imparte la conferencia de D. Federico Sopeña sobre "Beethoven, artista comprometido» y se desarrollan diversos conciertos entre los que cabe señalar: «Concierto para dos pianos» de Frechilla Zulcaga; "Concierto de piano» de Pilar Bayona; "Concierto de Clavecín» por Genoveva Gálvez.; concierto de la Orquesta de solistas de Madrid, dirigido Isido-

19 Información extraída de: AUSA, Memoria del año académico 1969/70, Universidad de Salamanca, 1971. Rev. 1178/41: 146.

20 Información extraída de: AUSA, Memoria del año académico 1970/71, Universidad de Salamanca, 1972. Rev. 1178/42: 174-175. 
ro García Polo; concierto del coro Alfonso el Sabio por José Luis Ochoa de Olza; concierto de piano por Carmen Vilá; concierto de los coros de la Universidad de Salamanca y de la de Oviedo; «Audición de la Pasión según S. Mateo» de J. S. Bach, presentada por Dámaso G. Fraile; concierto de piano por Martín Berkowitz y «Concierto de música del siglo xx». Solistas del grupo «Koan» de Madrid.

Asimismo, se inicia durante el mes de marzo de 1971 el Seminario de Historia de la música española, del que se ocupa Dámaso G. Fraile, encargado de la cátedra. La clausura del evento, el 22 de abril de 1971, corre a cargo de Gerardo Gombau.

El amplio y variado elenco de actividades desarrolladas durante este curso contrasta claramente con el reducido número de los dos cursos anteriores. Además de todo lo señalado, en el mes de mayo de 1971 se desarrolló la «Semana de música española», bajo el patrocinio de la DGBA del Ministerio de Educación y Ciencia y la Comisaría General de la Música. El programa del evento incluyó recitales de danza y violín por Mariemma y Agustín León Ara respectivamente; la actuación del cuarteto de Madrigalistas de Madrid, el quinteto de viento de la RTV española, los solistas de la Orquesta Sinfónica de RTE española, el Coro de la Escuela Superior de canto e, incluso, la Orquesta Nacional de España. Asimismo, se incluyó un recital de piano de Eduardo del Pueyo y un recital de canto por Victoria de los Ángeles.

Durante algunos de los días de celebración de esta "Semana de música española» se desarrolló un "Seminario sobre la educación musical» (días 5, 6 y 7 de mayo de 1971 en horario de 10.00 a 14.00 y de 17.00 a 19.00) que versó sobre las sociedades de conciertos en España.

Sin duda, estamos ante un curso académico de especial productividad en la Cátedra Francisco Salinas, con la organización de un elevado número de actividades, conciertos, cursos, audiciones, seminarios, etc. que demuestran su progresiva madurez e integración dentro de las actividades de extensión universitaria de esta institución.

\section{Curso $1971 / 72^{21}$}

Durante este año académico la cátedra desarrolla veinte conciertos, gestiona la actuación de cuatro orquestas (Orquesta de Cámara del Rhin, Colonia; «Detmolder Blaeserkreis», Alemania; Orquesta de Cámara de Sofía, Bulgaria; "The London Masterplayers Orchestra», Inglaterra), un coro (el de la Universidad de Salamanca), cuatro agrupaciones musicales de Cámara (Quinteto Clásico de R.T.V. española; Grupo L.E.M.A. de Madrid; «Gabrieli String Quartet» de Londres; y Trío «Foerster» de Praga) y cuatro pianistas (Jan Henrik Kayser, de Noruega; Zehaida Manfugás, de Cuba; Doris Rothmund, de Alemania; Haydee Helguera, de Argentina). Asimismo,

${ }^{21}$ Información extraída de: AUSA, Memoria del año académico 1971/72, Universidad de Salamanca, 1973. Rev. 1178/43: 151-152. 
se celebran conciertos de piano y violín, de guitarra, por Javier Quevedo, de París, de clavecín, por Frangoise Petit de París y un recital de Jazz por el grupo Play Hot Jazz. Sin duda, tras el repaso de los músicos y agrupaciones participantes, destaca en este curso la internacionalidad de las actuaciones desarrolladas.

Junto a la programación de conciertos, la Cátedra Salinas organiza un Curso de Formación Musical y diversos ciclos monográficos sobre Manuel De Falla (en el XXV aniversario de su muerte), Igor Strawinsky y J. S. Bach. Antes del inicio de las vacaciones navideñas se ofrece el "Weihnachtsoratorium» y, durante la época de Semana Santa, la «Pasión según San Mateo» de J. S. Bach.

Además, la cátedra de música salmantina inicia este curso una nueva actividad denominada como "Ciclo de intérpretes universitarios», dentro de la cual se engloban las actuaciones del grupo instrumental de la Universidad de Salamanca (28/01/1972 y $16 / 05 / 1972)$ y el concierto de piano E. Arnaltes, de la Universidad de Granada (28/04/1972).

Para terminar con el repaso a las actividades del curso, la cátedra ofrece dos actuaciones -concierto de piano y guitarra- el día 28 de enero de 1972 y un recital variado, el día 16 de mayo, con el objetivo de, tal y como se expresa en la propia memoria académica del curso, "fomentar la interpretación musical entre los alumnos de las diversas Facultades Universitarias» ${ }^{22}$.

\section{Curso $1972 / 73^{23}$}

Las actividades de la Cátedra Francisca Salinas siguen aumentando año tras año, llegando en este curso a la organización de un total de 27 conciertos (con pianistas como Rudolf Am Bachm de Zürich, Antotiio Baciero y Marisa Montiel; concierto de violonchelo y piano de Joaquín Vidaechea y M. a Jesús Aeches; de guitarra por Roberto Olabarrieta, Jaime Roberto Catalá y Narciso Yepes; o a la flauta y arpa, Jean-Claude Gerard y Chantal Mathieu, entre otros). En la memoria académica del curso 1972/73 podemos leer:

El primer objetivo de toda Cátedra de Música ha de ser el ofrecer en directo la obra artístico-musical, programar conciertos que sean el punto de contacto del oyente y del intérprete con la obra de arte. El número de conciertos ofrecidos, veintisiete -más de tres conciertos por mes- es un índice bien elocuente de la actividad de la Cátedra Salinas durante este Curso ${ }^{24}$.

22 Idem, Ibídem.

${ }^{23}$ Información extraída de: AUSA, Memoria del año académico 1972/73, Universidad de Salamanca, 1974. Rev. 1178/44: 199-203.

${ }^{24}$ Idem, p. 199. 
Intervienen en este curso las orquestas Ensemble Instrumental «Andrée Colson » de Tours; la Orquesta de Cámara de Madrid en homenaje a Gerardo Gombau, patrocinado por la Comisaría General de la Música, bajo la dirección de José M. Franco Gil y como soprano Isabel Penagos; la Orquesta de Cámara del Rhin, Colonia, con la dirección de Albert Kocsis; y la Orquesta del Palazzo Pitti, Florencia, bajo la dirección de Pietro Argento.

Se reciben este año en Salamanca a los siguientes coros y corales: Coral Polifónica Gijonesa (director: Carlos Sampedro); Coro de la Universidad de Salamanca (director: Jesús García Bernalt); Coro Alfonso X El Sabio, Madrid (director: José L. Ochoa de Olza); y Coro de la Universidad de Oviedo (director: Luis Gutiérrez Arias). En cuanto a pequeñas agrupaciones, nos encontramos con el «Trio Di Milano»; el Dúo pianístico de Javier Alfonso y M. a Teresa de los Ángeles; y el Cuarteto Clásico de R.T.V. española (concierto homenaje a D. Aníbal Sánchez Fraile).

La celebración del III Ciclo de intérpretes españoles en España (organizado por la Comisaría General de la Música de la DGBA) trae los siguientes conciertos: a) Viola y piano: Enrique Santiago y Ana M. a Gorostiaga; b) Dúo pianístico: FrechillaZuloaga; c) Clarinete y piano: Jesús Villa Rojo y Elisa Ibáñez.

Nuevamente, la cátedra organiza un recital de jazz con la banda «The Millikin Jazz Lab Band» de la Universidad de Millikin (EE. UU.). Y también un recital de canto con la sesión de Joaquín Díaz y la de María Viñals, Soprano. A ello se suma la organización de un Ciclo de intérpretes universitarios.

Además del elenco de actividades y conciertos musicales reflejados en el anterior listado, la cátedra imparte el Curso de Formación Musical, a cargo del encargado de la cátedra, Dámaso García Fraile y una serie de profesores invitados (Tomás Marco, Ramón Barce, Enrique García Asensio y Enrique Llacer «Regolí»). En él participan la nada desdeñable cifra de 123 alumnos de distintas facultades salmantinas y se imparten un total de 23 conferencias, conciertos y audiciones, entre las que cabe señalar, a modo de ejemplo, las siguientes: La evolución de la música española; El Barroco Tardío en Italia: Los clavecinistas franceses. La música instrumental en España durante los siglos XVII-XVIII; Gerardo Gombau, compositor de Vanguardia; Música y sociedad en la época de la Ilustración; La música instrumental postbeethoveniana. La sinfonía y el poema sinfónico. El oratorio. La música francesa después de Berlioz; La dirección de orquesta a través de los tiempos. El director, su personalidad y sus problemas; La música española contemporánea; o La música serial: Schoenberg, Berg y Webern. 
El nuevo año académico sirve a la cátedra de música salmantina para poner en marcha algunas actividades novedosas. Así, por ejemplo, en colaboración con el Departamento de Germanística se celebra un ciclo de Ópera Alemana con filmaciones en color de la Opera de Hamburgo: "Las Bodas de Fígaro» de W. A. Mozart (09/03/1974); "La flauta mágica» de W. A. Mozart (11/11/1974); «El cazador furtivo» de C. M. von Weber (12/11/1974); "Los Maestros cantores de Nurember», 1. parte de R. Wagner (13/11/1974); «Los Maestros cantores», 2. a parte (14/11/1974); «Wozeck» de Alban Berg (15/11/1974).

También se desarrolla un ciclo sobre Música de Jazz del que se dice en la memoria académica del año que "tuvo gran aceptación entre los estudiantes universitarios ${ }^{26}$. La parte teórico-práctica de este ciclo estuvo a cargo de Pedro Iturralde, con la conferencia "La música de Jazz, su evolución melódica», José María Martín Porrás, con «Evolución de la percusión. La rítmica del Jazz» así como un recital de percusión, y José Vida, que se encargó de una audición de música de jazz con una selección de obras y presentación. Asimismo, se ofrecieron dos recitales de Jazz en el Teatro Coliseum de la ciudad, uno de ellos con la intervención de Vladimiro Bas, Joey Mercer, Carlos Villa, Dave Thomas y José Nieto, y otro de clausura del ciclo sobre «Traditional jazz studio de Praga» bajo la dirección de Pavel Smetacek.

Nuevamente se llevó a cabo el Curso de Formación Musical, incrementándose la matrícula en esta ocasión hasta los 190 alumnos, llegándose a registrarse en alguna de las sesiones abiertas al público hasta 250 participantes. En total se impartieron 23 conferencias, algunas de ellas a cargo del encargado de la cátedra, Dámaso G. Fraile, y las restantes actuando como profesores invitados: Cristóbal Halffter, Federico Sopeña y Joaquín Rodrigo.

El número de conciertos celebrados continúa situándose en números similares a los años anteriores. Como muestra de ellos, cabe señalar el concierto de piano por Franz F. Eichberger; el concierto de guitarra y violín, por Patrice Fontanarosa y Minchel Dintrich; el concierto de los "Solistas de Filadelfia» (The Philadelphia Chamber soloists); el concierto de trompeta y órgano (desarrollado en la Iglesia del Colegio Fonseca) con André Bernard a la trompeta y Edgar Krapp al órgano; el concierto de la orquesta "Camerata», de Bucarest (director: Paul Staicu); el concierto de órgano por Antoine Sibertin-Blanc o el concierto de la orquesta «The Academie of St. Martin In-Thefields», patrocinado por la Fundación Juan March.

Crecen especialmente en este curso las conferencias impartidas a los universitarios, mayoritariamente dictadas por el encargado de la cátedra. Entre los nu-

25 Información extraída de: AUSA, Memoria del año académico 1973/74, Universidad de Salamanca, 1975. Rev. 1178/45: 161-165.

${ }^{26}$ Idem, p. 161. 
merosos títulos podemos señalar aquí, por ejemplo, La Música en la sociedad del Renacimiento; La Música del estilo barroco. Características; El barroco tardío en la música europea; Estudio de la obra de J. S. Bach; o El impresionismo.

Durante el mes de abril de 1974 se desarrolla nuevamente la Semana de Intérpretes Universitarios con un recital de música Folk y otro del grupo "Al-Andalus», un concierto de piano, guitarra y música de cámara y otro concierto de guitarra, un recital de solistas y la interpretación de A Nazim Hikmet, «Tlaloc». Durante este mes se desarrolla también un recital de música «Bluegrass» titulado The Mclain Family Singers, patrocinado por la Asociación Cultural Hispano-Norteamericana.

\section{Curso $1974 / 75^{27}$}

Llegamos así al último año académico marcado en este trabajo como marco de estudio y nos encontramos con la organización de 25 conciertos a través de la cátedra Francisco Salinas. Como novedades, y gracias al apoyo de la Dirección General de Universidades e Investigación, se pone en marcha el ciclo «Música en la Universidad», inaugurado con el concierto de guitarra de Narciso Yepes en el mes de octubre de 1974. En meses sucesivos actuarían la orquesta de Cámara «Paul Kuentz» y la orquesta de Cámara de Varsovia, el pianista de Rafael Orozco, el Cuarteto Beethoven de Roma, el violinista Salvatore Accardo, el cantor José Menese y la orquesta francesa de J. F. Paillard.

Asimismo, destaca la puesta en marcha de la nueva Sociedad de Conciertos de Salamanca, integrada por habitantes de la ciudad y estudiantes de su universidad. En nota de prensa publicada por el Rectorado durante el mes de octubre se decía lo siguiente:

La Universidad tomó parte activa desde el principio en la estructuración de la nueva Sociedad de Conciertos. Como quiera que las posibilidades de programación que ofrece dicha Sociedad son más amplias que las que pueda realizar por sí sola la Cátedra Salinas, este Rectorado ha decidido colaborar con ella, subvencionando la asistencia de los alumnos universitarios a los conciertos que se programan. Ha quedado reservado un número de localidades semejante al aforo del Aula Juan del Enzina. Los alumnos de esta Universidad pueden disponer de ellas al cincuenta por ciento de su precio normal ${ }^{28}$.

La Sociedad de Conciertos ofreció un total de 16 actos, en el Teatro Coliseum, asistiendo a cada uno de ellos un gran número de estudiantes. Los más destacados fueron: Orquesta «Leos Janacek»; Dúo de pianos «Billard-Azais»; concierto de

27 Información extraída de: AUSA, Memoria del año académico 1974/75, Universidad de Salamanca, 1976. Rev. 1178/46: 164-165.

${ }^{28}$ Idem, Ibídem. 
Violoncelo y piano «Aldulescu-Soler»; concierto de piano por «V. Afanassier»; orquesta de Cámara Rumana de Gluj; Camerata de Bucarest; Traditional Jazz Studio de Praga; Ballet eslovaco «Sluk» y orquesta Filarmónica del Estado de Kosice (director Mario Klemens, violinista Jela Spitkova).

Se celebraron cuatro conciertos pertenecientes al V Ciclo de intérpretes españoles en España, patrocinado por la Dirección General del Patrimonio artístico y cultural del Ministerio de Educación y Ciencia. Comisaría Nacional de Música: Concierto de arpa por M. a Rosa Calvo Manzano; Concierto de violín y piano por Francesch-Besses; Recital de canto y piano por Ohrt-Peris y Cuarteto «Atríum Musícae».

La Cátedra Salinas, además, celebró un «Homenaje a Schonberg», en el primer centenario de su nacimiento, con un concierto de piano a cargo de Pedro Espinosa. Isidro Barrio dio un concierto de piano y, en colaboración con el Departamento de francés, se celebró un concierto de viola y piano por la Srta. Witterkoer y la Sra. Thomas.

Durante el mes de febrero, tuvo lugar la II Semana de intérpretes universitarios, en la que actuaron, durante ocho días, varios grupos y solistas de la Universidad de Salamanca. Asistieron unos cuatro mil espectadores. Igualmente, la Cátedra Salinas colaboró con el Aula cultural Anue en la programación de la «Semana de Música Folk».

En esta ocasión, el Curso de Formación Musical se dedicó al estudio de «La Música durante los cien últimos años» impartiéndose, en total, 25 lecciones a cargo de D. Dámaso García Fraile. Actuaron como profesores invitados: Ramón G. Amezúa, tesorero de la Academia de Bellas Artes de San Fernando; Agustín G. Acilu, compositor, y el flamencólogo Francisco Almazán. En este curso la matrícula se situó en los 154 alumnos.

A modo de conclusión, a lo largo del periodo analizado se percibe un notable incremento en el número de actividades organizadas por la cátedra. Cabe destacar, más allá de la ampliación progresiva de la oferta, la voluntad manifiesta por parte de los responsables de incluir propuestas de calidad en toda la gama de actividades desarrolladas. Ya en el primer año, sin ser muy prolífico en propuestas, encontramos la presencia de figuras de reconocido prestigio nacional e internacional, como la pianista Marisa Montiel. En la actividad concertística de los años sucesivos se siguió optando por intérpretes y agrupaciones de renombre y casi siempre en formato solista o pequeños grupos de cámara. Eran recurrentes los recitales de piano, bien fuere como solista o bien acompañando a cantantes u otros instrumentos, conciertos con pequeñas agrupaciones de cuerda o viento, orquesta de cámara, coros, grupos de jazz, etc. Cabe remarcar, aunque en menor medida, la apuesta por repertorios y formaciones menos frecuentes como las integradas por trompeta y órgano, violín y guitarra o flauta y arpa. Destacamos la presencia, entre otros, de pianistas reconocidas como Lily Bienvenu, Pilar Bayona o Carme Vilà, el violinista Agustín León Ara, el guitarrista Regino Sainz de la Maza, la clavecinista Genoveva Gálvez, la cantante Victoria de los Ángeles; o agrupaciones como el Coro Universitario de Oviedo, Madrigalistas de Praga, Coro Alfonso X El Sabio de Madrid, Atrium 
Musicae, Cuarteto Beethoven de Roma, Orquesta de RTE española, Orquesta de solistas de Madrid, Orquesta Nacional de España, Orquesta del Rhin, The London Masterplayers Orchestra, The Millikin Jazz Lab Band, etc.

Paralelamente a la actividad concertística se llevaron a cabo desde la Cátedra Francisco Salinas un gran número de audiciones, conferencias, cursos y seminarios. Las audiciones abarcaron un largo abanico de periodos y estilos, predominando las de autores románticos y contemporáneos. Dichas audiciones contaban generalmente con la presencia de destacados músicos, musicólogos o estudiosos de la historia y la estética musicales que aportaban un valor añadido a la audición. Entre otros, destacamos a Dámaso García Fraile, Federico Sopeña, Pedro Iturralde, Gerardo Gombau, Tomás Marco, Enrique García Asensio, Cristóbal Halffter o Joaquín Rodrigo. Además de todo lo señalado, y en la línea del fomento de la práctica musical entre los universitarios, debe remarcarse también la creación del «Ciclo de intérpretes universitarios».

\section{CONCLUSIONES}

Las cátedras especiales de música suponen un hito en cuanto a la introducción de la música en la universidad de la época, pero siempre entendida esta como actividad de extensión universitaria y de difusión cultural, todavía muy lejos de la concepción de la necesaria introducción de este tipo de estudios, con entidad propia, dentro de las facultades. Abren la puerta a concertistas y músicos tanto nacionales como internacionales, consiguiéndose de este modo la penetración y difusión de la cultura musical de dentro y fuera del país en los espacios universitarios. A pesar de ello, obviamente, el reducido público al que llegaban el tipo de actividades organizadas por las cátedras especiales limitaba el alcance de este positivo aspecto.

Asimismo, las nuevas cátedras especiales de música desarrollan una encomiable labor en relación a la creación de archivos, recopilación y edición de composiciones, obras o estudios de cultura musical de interés artístico, científico o histórico, y diversas acciones con las que, en esencia, se pretendía recuperar, guardar y poner a disposición del investigador o interesado parte de la cultura popular, en relación a tradiciones musicales, del país. Hay, por tanto, un evidente interés en la conservación de la cultura, al mismo tiempo que se busca su difusión mediante la edición de obras y composiciones. Si todas las cátedras mencionadas en este estudio realizaron de forma efectiva una labor de este tipo, probablemente ayudaron a que, posteriormente, con la introducción de los estudios de Musicología en las universidades, se dispusiera ya de un importante material para la elaboración de trabajos, tesis doctorales, etc.

Por otra parte, las composiciones de los patronatos o comisiones previstas para el buen funcionamiento de las cátedras integraron a miembros de corporaciones locales y 
municipales -representantes de ayuntamientos, diputaciones provinciales, conservatorios, sociedades filarmónicas, etc.--, aspecto del todo necesario para iniciar el camino de acercamiento entre universidad y sociedad, y para hacer efectivo el concepto de participación, en una época en la que la universidad se encontraba parcialmente aislada de los retos sociales más acuciantes y, al mismo tiempo, de una sociedad ajena y distanciada en su conjunto de todo aquello que se hacía dentro de la universidad.

En el caso concreto de la Cátedra Francisco Salinas, a través de su actividad concertística, las audiciones organizadas, los cursos y seminarios especializados, y, finalmente, el "Ciclo de intérpretes universitarios», transmitió una clara idea de divulgación y culturización musical de la comunidad universitaria. Si bien esta aportación se llevó a cabo desde un ámbito extracurricular, sin vinculación alguna con los planes de estudios vigentes, a bien seguro contribuyó, en mayor o menor medida, a la reimplantación posterior de los estudios de música en la universidad. Por otra parte, la cátedra charra tomó parte activa en la creación de la Sociedad de Conciertos de Salamanca, aspecto remarcable en el ya mencionado necesario acercamiento entre universidad y ciudad.

En todo caso, resulta necesario apuntar aquí que el trabajo presentado supone el primer peldaño de un recorrido más amplio. Quedan abiertas líneas de investigación en relación, por ejemplo, al estudio comparado de la actividad real ejercida por estas cátedras dentro de las distintas universidades o el contraste de estos datos con testimonios orales que participaran o tomaran parte de las actividades de estas cátedras.

En conclusión, consideramos que estas cátedras, siempre y cuando sean entendidas dentro de su contexto e insertas en el ámbito de la extensión universitaria, ayudaron en cierta medida a la posterior aparición de los estudios universitarios de musicología. Es obvio que podría haberse hecho mucho más por la música dentro de la universidad y que, de algún modo, la creación de estas cátedras supuso un "parcheado" sobre una situación que debía de cambiar, pero, después de realizar un estudio de sus actividades, circunscrito en todo caso a la Universidad de Salamanca, no puede negarse que su labor resulta destacable.

\section{REFERENCIAS BIBLIOGRÁFICAS}

Alonso Medina, J. A., Rodríguez Cruz, P. (1999). Evolución histórica de la formación musical de los maestros (1900-1967). Boletín Millares Carlo, 18, pp. 87-105.

Aviñoa, X. (Ed.) (1996). Miscel/lània. Oriol Martorell. Barcelona: Publicacions de la Universitat de Barcelona.

Carpenter, N. C. (1972). Music in the Medieval and Renaissance Universities. New York: Da Capo Press. 
Casares Rodicio, E. (1986). Presente y futuro de la música en la Universidad española. En Actas del I Simposio Nacional de Música (pp. 153-160). Madrid.

Casares Rodicio, E. (1996). Memoria de un reencuentro. La música en la universidad. En X. Aviñoa (Ed.), Miscel·lània. Oriol Martorell (pp. 27-34). Barcelona: Publicacions de la Universitat de Barcelona.

García-Bernalt Alonso, B. (2014). Música y fiesta religiosa en la Universidad de Salamanca en la Ilustración. Neuma. Revista de música y docencia musical, 7, pp. 10-39.

García-Bernalt Alonso, B. (2015). La música y su entorno en la Universidad de Salamanca del Siglo de las Luces: las fronteras de un caso periférico. Neuma. Revista de música y docencia musical, 8, pp. 11-47.

García Fraile, D. (1991). La cátedra de música de la Universidad de Salamanca durante diecisiete años del siglo XV (1464-1481). Anuario musical, 46, pp. 57102.

García Fraile, D. (1999). La cátedra de música de la Universidad de Salamanca. En Historia de la Universidad de Salamanca (pp. 237-267). Salamanca: Ediciones Universidad de Salamanca

García Fraile, D. (2000). La vida musical en la Universidad de Salamanca durante el siglo XVI. Revista de Musicología, 23(1), pp. 9-74. https://doi. org/10.2307/20797633.

García Fraile, D. (2004). La música en la Universidad de Salamanca. En Congreso Internacional Música y Universidad (pp. 29-71). Salamanca: Departamento de Didáctica de la expresión musical, plástica y corporal.

Giménez Martínez, J. J. (2000). La extensión universitaria en España. En V Congreso Iberoamericano de Extensión. Sociedad, Educación Superior y Extensión: Balance y Perspectivas. México: Universidad Michoacana de San Nicolás de Hidalgo.

Hernández Díaz, J. M.a (2011). El modelo de la universidad alemana en España. En J. M. a Hernández Díaz (coord.), La pedagogía alemana en España e Iberoamérica (1810-2010) (pp. 225-247). Salamanca: Castilla Ediciones.

Marías, Á. (1999). ¿La música en la Universidad? Cuenta y razón, 112, pp. 76-79. 
Martín Moreno, A. (2005). Pasado, presente y futuro de la Musicología en la Universidad española. Revista interuniversitaria de formación del profesorado, 19(1), pp. 53-76.

Martín Moreno, A. La presencia histórica de la Música en la Universidad Española y los retos de los Conservatorios Superiores en su integración universitaria. MAR - Música de Andalucía en la Red, n.o extraordinario 4, pp. 28-34.

Medina, A. (1986). El esperado retorno de la música a la universidad española. Revista Ritmo, 562, pp. 14-15.

Menéndez Pelayo, M. (1974). La estética en los tratadistas de música durante los siglos XVI y XVII. Historia de las Ideas Estéticas en España (1883-1891). Madrid: CSIC, 4. a ed., tomo I.

Muñiz Velázquez, J. A. (1998). La Música en el Sistema Propagandístico Franquista. Historia y Comunicación Social, 3, pp. 343-363.

Oriol De Alarcón, N. (2012). Contribución de la enseñanza musical, en los estudios de Magisterio en España, a la conservación del arte y la cultura popular. DEDICA. Revista de Educaçao e Humanidades, 3, pp. 13-42.

Piñeiro Blanca, J. (2013). Instrumentalización política de la música desde el franquismo hasta la consolidación de la democracia en España. Revista del CEHGR, 25, pp. 237-262.

Salinas, F. (2013). De Musica libri septem [edición al cuidado de Amaya García Pérez y Bernardo García-Bernalt Alonso]. Salamanca: Ediciones Universidad Salamanca.

Sarget Ros, M. a Á. (2000). Perspectiva histórica de la educación musical. Ensayos: Revista de la Facultad de Educación de Albacete, 15, pp. 117-130.

Tünnermann Bernheim, C. (2003). La Universidad ante los retos del siglo XXI. México: Ediciones de la Universidad Autónoma de Yucatán.

Tur Mayan, P. (1992). Reflexiones sobre la Educación Musical. Historia del pensamiento filosófico musical. Barcelona: Universitat de Barcelona Publicacions.

Wolf, J. (1965). Historia de la Música. Barcelona: Editorial Labor. 